\title{
A fast edge charge eXchange recombination spectroscopy system at the ASDEX
}

\section{Upgrade tokamak}

\author{
M. Cavedon, ${ }^{1,2, a)}$ T. Pütterich, ${ }^{1}$ E. Viezzer, ${ }^{1,3}{ }^{3}$ R. Dux ${ }^{1}$ B. Geiger, ${ }^{1}$ R. M. McDermott, ${ }^{1}$ \\ H. Meyer, ${ }^{4}$ U. Stroth, ${ }^{1,2}$ and the ASDEX Upgrade Team ${ }^{1,}$ b) \\ 1) Max-Plank-Institut für Plasmaphysik, D-85748, Garching, \\ Germany \\ 2) Physik-Department E28, Technische Universität München, 85748 Garching, Germany \\ ${ }^{3)}$ Dept. of Atomic, Molecular and Nuclear Physics, University of Seville, \\ Avda. Reina Mercedes, 41012 Seville, Spain \\ 4) EURATOM/CCFE Fusion Association, Culham Science Centre, Abingdon, Oxon, \\ $O X 143 D B, U K$
}

(Dated: Tuesday $8^{\text {th }}$ August, 2017)

In this work, a new type of high through-put Czerny-Turner spectrometer has been developed which allows to acquire multiple channels simultaneously with a repetition time on the order of $10 \mu \mathrm{s}$ at different wavelengths. The spectrometer has been coupled to the edge charge exchange recombination system at ASDEX Upgrade which has been recently refurbished with new lines of sight. Construction features, calibration methods, and initial measurements obtained with the new setup will be presented.

\footnotetext{
a) Electronic mail: marco.cavedon@ipp.mpg.de

b) For authors' list, see H. Zohm et al., NF 55104010 (2015)
} 


\section{INTRODUCTION}

Charge exchange recombination spectroscopy (CXRS) is a powerful method for measuring spatially resolved ion temperatures $T_{i}$, impurity densities $n_{\alpha}$ and velocities $v_{\alpha}$ in fusion plasmas $^{1}$. The basic principle of CXRS exploits spectral lines emitted after charge exchange from neutral atoms into highly excited states of impurity ions. The subsequent decay of the excited state of the impurity ion leads to the emission of a photon at a specific wavelength. Thus, the ion temperature and the flow velocity are obtained from the Doppler width and Doppler shift and the impurity density from the radiance of the emission line. For magnetized fusion plasmas, these measurements can be combined in the radial force balance equation to determine the radial electric field $E_{r}^{2}$.

In the past years, the ASDEX Upgrade tokamak (AUG) has been equipped with several charge exchange systems viewing the core and the edge of the plasma and providing tempo-

rally and spatially resolved CXRS profiles ${ }^{3 / 4}$. Each system is connected to a high-throughput Czerny-Turner spectrometer equipped with charge-coupled device (CCD) cameras. So far, the minimum time integration of the systems was $2.3 \mathrm{~ms}$ which is barely adequate to study phenomena like edge localized modes (ELMs) and definitely insufficient to address the evolution of $E_{r}$ throughout the L-H transition. Several solutions have been developed, along the years, to reach sub-millisecond CXRS measurements in fusion plasmas ${ }^{59}$. Although some of them could reach a temporal resolution of even $1 \mu \mathrm{s}$, they are often limited to one or two channels per spectrometer and/or to a fixed wavelength range. Here, a modified version of a typical Czerny-Turner spectrometer coupled with an electron multiplying (EM) CCD camera is proposed which allows fast CXRS measurements down to $10 \mu$ s of time resolution for up to nine channels at different wavelengths.

\section{SPECTROMETER DESIGN}

The design choices developed in this work were taken to improve the temporal resolution of a conventional Czerny-Turner spectrometer preserving the flexibility of a turnable grating, e.g. changeable wavelengths, and multiple acquirable channels. Moreover, the new system should feature similar wavelength dispersion $\Delta \lambda /$ pixels of approximately $0.27 \AA /$ pixel at $500 \mathrm{~nm}$, in order to resolve velocities of 1 to $2 \mathrm{~km} / \mathrm{s}$ which correspond to roughly $1 / 10$ of 
(a) Conventional

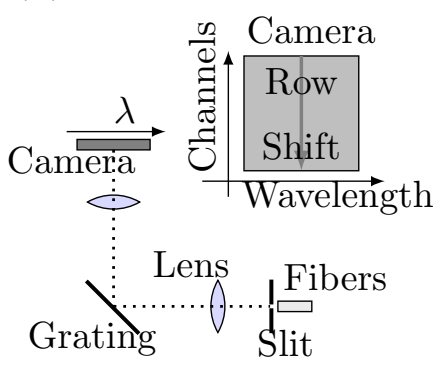

(b) New design

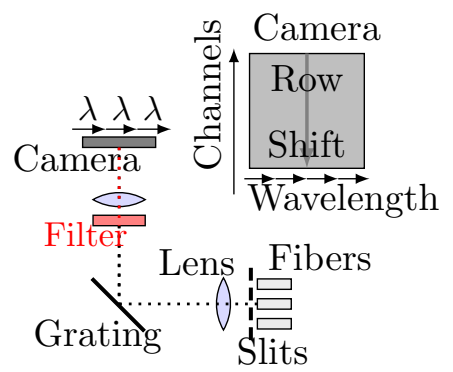

FIG. 1. (a) Schematic of a conventional Czerny-Turner spectrometer with a single slit. (b) Schematic of the adapted Czerny-Turner spectrometer for fast measurements. Several slits are installed at the spectrometer entrance to image multiple channels on a smaller camera chip area. The interesting part of the spectra is then selected by an interference filter (red) positioned before the focussing lens.

a pixel at $500 \mathrm{~nm}$. Such a shift is detectable given the ansatz of a Gaussian line emission. The resolution of lower velocities is prevented by uncertainties arising from atomic physics

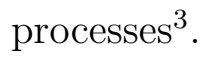

The principle of a conventional Czerny-Turner spectrometer is illustrated in figure 1a. The light entering the entrance slit is collimated through a lens onto a turnable grating and re-focussed on a camera by a second lens. Multiple channels can be imaged simultaneously by arranging the fibers vertically along the entrance slit. In this way, the spectra of the different fibers, e.g. the wavelength axis, are not overlapping on the camera chip and therefore can be acquired separately. On ASDEX Upgrade, such spectrometers are usually equipped with a variable slit typically set to 50 or $100 \mu \mathrm{m}$, two objective lenses (Leica APO-ELMARIT$\mathrm{R}^{10}$ ) with each a focal length of $280 \mathrm{~mm}$ (collimating lens) and $180 \mathrm{~mm}$ (focussing lens), respectively, and a movable grating with 2400 grooves/mm. The lenses are chosen such that, due to the demagnification, twenty-five fibers with a diameter of $400 \mu \mathrm{m}$ can be imaged onto a Princeton Instruments ProEM charge coupled device (CCD) camera ${ }^{11}$ with on-chip multiplication gain featuring a light sensitive area with $512 \times 51216 \mu \mathrm{m}$ pixels.

The temporal resolution of the system is limited by the camera repetition time. Its minimum $\Delta t_{\min }$ can be calculated as follows:

$$
\Delta t_{\mathrm{min}}=\frac{n_{\mathrm{px}, \mathrm{row}} \cdot n_{\mathrm{ch}}}{f_{\mathrm{ro}}}+n_{\mathrm{l}} \cdot v_{\mathrm{sh}}+\Delta t_{\mathrm{cam}}
$$

where $n_{\mathrm{px}, \text { row }}$ is the number of pixel per channel, $n_{\mathrm{ch}}$ the number of channels, $f_{\text {ro }}$ the read- 
out frequency, $n_{\mathrm{l}}$ the number of lines to shift, $v_{\mathrm{sh}}$ the shift speed and $\Delta t_{\text {cam }}$ eventual delays due to the camera processing such as the application of the pixel bias correction (PBC) or the cleaning of serial registers. For the old setup, e.g. for $n_{\mathrm{px} \text {,row }}=512, n_{\mathrm{ch}}=25$, $n_{\mathrm{l}}=512+40$ (Mask), $v_{\mathrm{sh}}=0.45 \mu \mathrm{s} / \mathrm{row}, f_{\mathrm{ro}}=10 \mathrm{MHz}$, the minimum acquisition time is equal to $\Delta t_{\text {min,old }}=2.27 \mathrm{~ms}$. Hence, it is clear that given a certain camera, e.g. for fixed $f_{\mathrm{ro}}, v_{\mathrm{sh}}$ and $\Delta t_{\text {cam }}$, one needs to reduce $n_{\mathrm{px}}$ and $n_{\mathrm{l}}$ to improve the temporal resolution. Since the interesting part of the spectra only occupies between 10 and $20 \%$ of the entire camera chip, both $n_{\mathrm{px}}$ and $n_{\mathrm{l}}$ can be reduced by rearranging the imaged spectra on the camera chip. To do this, a re-design of the conventional Czerny-Turner spectrometer is required.

Figure $1 \mathrm{~b}$ shows a schematic of the modified Czerny-Turner spectrometer developed in this work. An interference filter (in red) has been installed between the grating and the focussing lens in order to select only the interesting part of the spectrum. This way several channels can be arranged along the wavelength axis $(\lambda)$ perpendicular to the read-out direction without overlapping between neighbouring spectral lines. To that end, nine fixed slits, each $50 \mu \mathrm{m}$ wide, have been installed at the spectrometer entrance which allow to acquire several channels simultaneously on a single chip row. Note that the use of multiple parallel slits combined with a band-pass interference filter has already been adopted in previous works, in general to increase the number of acquirable channels ${ }^{12113}$. In order to keep the full flexibility of using the spectrometer in the conventional way, the middle slit can accommodate 25 channels as for the design in figure $1 \mathrm{a}$ while the other eight slits only two. In figure $2 \mathrm{a}$ an overview of the fixed entrance slit system is shown while in figure $2 \mathrm{~b}, \mathrm{c}$ the details of the bottom-left slit are highlighted. The parallel slits are aligned at one end of the long central slit in order that their projected images are positioned at the bottom end of the camera chip, close to the read-out region, with the purpose of avoiding unnecessary row shifts.

The dispersion $\partial \lambda / \partial \mathrm{x}$, where $\lambda$ is the wavelength and $x$ the real space on the camera chip, of the Czerny-Turner spectrometer is a function of the wavelength ${ }^{14}$ and it decreases by increasing wavelength. Moreover, for a certain temperature $\Delta \lambda / \lambda$ is a constant and therefore for longer wavelengths $\Delta \lambda$ increases. Hence the number of possible channels which can be imaged without overlapping onto the camera chip depends on the wavelength. For instance, in case of the helium CX-line $\mathrm{He}^{2+}(\mathrm{n}=4 \rightarrow 3)$ at $\lambda=468.571 \mathrm{~nm} 9$ channels can be simultaneously acquired while for the fully ionized nitrogen CX-line $\mathrm{N}^{7+}(\mathrm{n}=9 \rightarrow 8)$ positioned 


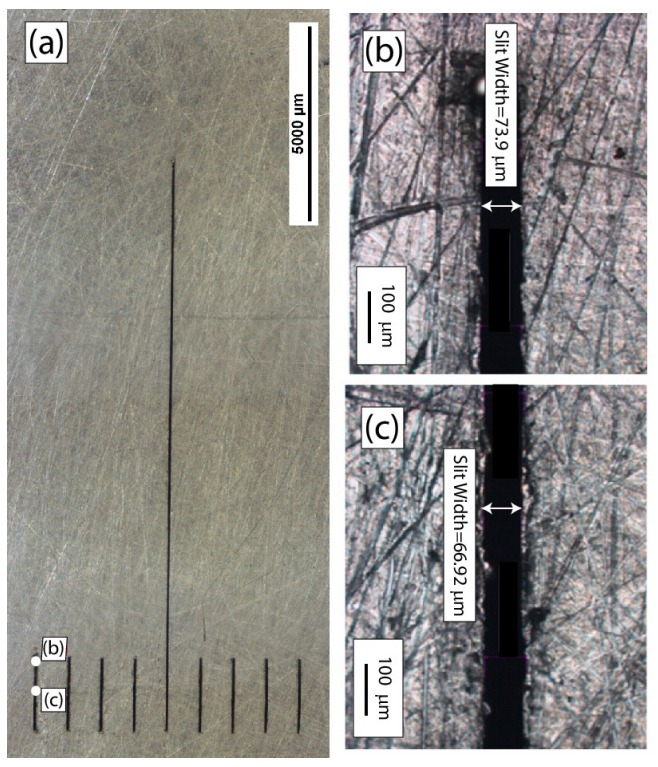

FIG. 2. Entrance slits of the fast Czerny-Turner like spectrometer. (a) Overview of the entire entrance slits; (b,c) Details of bottom-left slit where local measurements of the slit width are reported in $\mu \mathrm{m}$.

at $\lambda=566.937 \mathrm{~nm}$ only 5 channels can be imaged on the chip without overlapping. The $\mathrm{He}^{2+} \mathrm{CX}$-line is particularly suitable for this application since, in typical AUG discharges, it does not present any surrounding line within a range of roughly $6 \mathrm{~nm}$. Hence, the band-pass of the interference filter does not need to be too narrow making its usage easier.

\section{CCD CAMERA ACQUISITION MODES}

CCD cameras commonly used for high duty-cycle applications feature frame-transfer chips. They use a two-part sensor $512 \times 1040$ in which one-half of the chip is used as a storage area and is protected from light. The incoming photons are instead collected on the image area which, after being exposed, is rapidly shifted to the storage area. While the image area is exposed with light, the stored charges are readout. Thus, high repetition measurements are achieved avoiding dead times due to the chip read-out. A schematic of the frame-transfer CCD camera sensor is shown in figure 3 a.

Due to the re-design of the Czerny-Turner spectrometer reported in section II, the acquisition mode of the CCD camera can be optimized to reach high repetition rates. The vertical image of a fiber due to the demagnification of the lenses covers roughly an area 

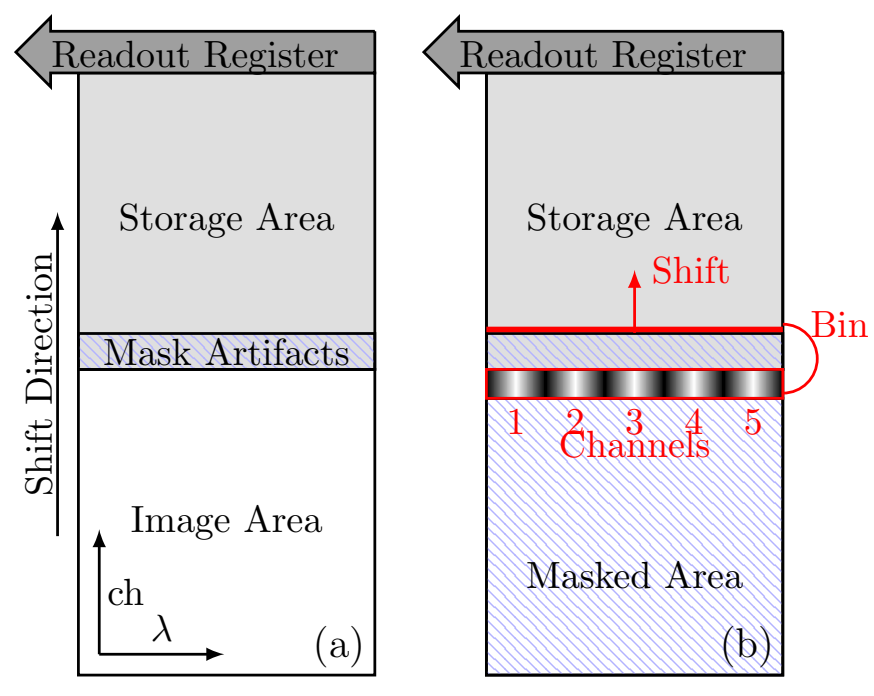

FIG. 3. (a) Schematic of frame transfer CCD camera. The sensor is divided in two parts: the image area where the incoming photons are focussed and the storage area where the integrated charges are shifted before the read-out. (b) Working principle of "Spectra Kinetics" acquisition mode developed by Princeton Instruments $\frac{11}{11}$. The incoming photons are focussed only on a certain number of rows (highlighted in red) which are shifted and binned onto the first row of the storage area. The process is repeated until the storage region is filled, which is then read-out interrupting the binning and shifting process, i.e. leading to dead time of approximately $30 \mathrm{~ms}$.

of 20 pixels and using the interference filter, multiple channels can be imaged on the same rows. Thus, the maximum repetition time using the standard frame transfer acquisition mode can be reduced to $0.71 \mathrm{~ms}$ for up to 9 channels. A further increase of the temporal resolution is obtained by reducing the parallel chip size (this mode is often called custom chip mode). Skipping the serial register cleaning and not applying the PBC, the maximum repetition time is $0.22 \mathrm{~ms}$ for 38 vertical rows. For the Princeton Instruments ProEM camera, the custom chip mode can only be applied following precise rules in order to not interfere with the GigE Data Transfer requirements (specifications can be found in ${ }^{11}$ ). While this acquisition mode reduces the repetition time by one order of magnitude compared to the standard Czerny-Turner spectrometer, a further improvement can be obtained by exploiting the capability of such cameras to shift row-arrays on a sub-micro-second timescale.

Figure 3b shows the working principle of the "Spectra Kinetics" acquisition mode developed by Princeton Instruments 11 . Here, after exposure a certain number of rows $n_{r}$ (highlighted in red), are binned together at the edge of the image area after being exposed 
with light transferred into the first row of the storage region. This process is extremely fast; if the rows, on which the light is focussed, are just at the edge storage area then the repetition time is equal to $\Delta t=\left(n_{r}+n_{\text {mask }}\right) \cdot v_{\mathrm{sh}}$, where $n_{\text {mask }}$ is the number of rows between the image and storage regions. In case of the ProEM camera used in the present work, $n_{\text {mask }}=10$ and hence $\Delta t=(20+10)$ rows $\cdot 0.45 \mu \mathrm{s} /$ rows $=13.5 \mu \mathrm{s}$. This can be further reduced down to $\approx 5 \mu \mathrm{s}$ by imaging just a part of the fiber onto the camera chip or by further demagnifying the fiber image. Note that the remaining part of the image region need to be masked from light to avoid interference with the acquired spectra. Moreover, the shift time through the masked region between the storage and image area needs to be taken into account in the calculation of the measurement time point and of the effective exposure time. The process of binning and shifting can be repeated only until the storage area is filled, i.e. 528 times in case of the ProEM camera used here. Afterwards, the entire chip has to be read out such that a new burst of exposures can start. The reading out of the entire storage region lasts $(512 \cdot 528) \cdot f_{\mathrm{RO}} \approx 30 \mathrm{~ms}$. Hence, this acquisition mode is intrinsically operating in a burst mode featuring dead times.

To summarize, the best performance for continuous acquisition is obtained via the custom chip mode with a minimum repetition time of $220 \mu \mathrm{s}$. The spectra kinetic mode allows measurements down to $5 \mu \mathrm{s}$ but it operates in a burst mode with roughly $30 \mathrm{~ms}$ of dead time.

\section{SPECTROMETER CALIBRATION}

The interpretation of the CXRS measurements needs an accurate wavelength and intensity calibration. Wavelength calibrations are required for velocity measurements and the intensity calibration of the entire system (in-torus optical heads, connecting fibers, spectrometer and camera) is necessary for determining the impurity density. Moreover, the instrument function of the system has to be characterized to deconvolve the Doppler broadening from the imperfections of the spectrometer optics and to measure the ion temperature.

During wavelength calibration the free parameters for the calculation of the wavelength axis ${ }^{14}$, i.e. the central wavelength $\lambda_{0}$, the focal length $f_{2}$, the vertical offset of the optical axis due to eventual misalignment $\delta h$ and the offset of every fiber from the central position $\delta x_{\mathrm{px}}$, are determined. Details of the derivation of the wavelength axis of Czerny-Turner 

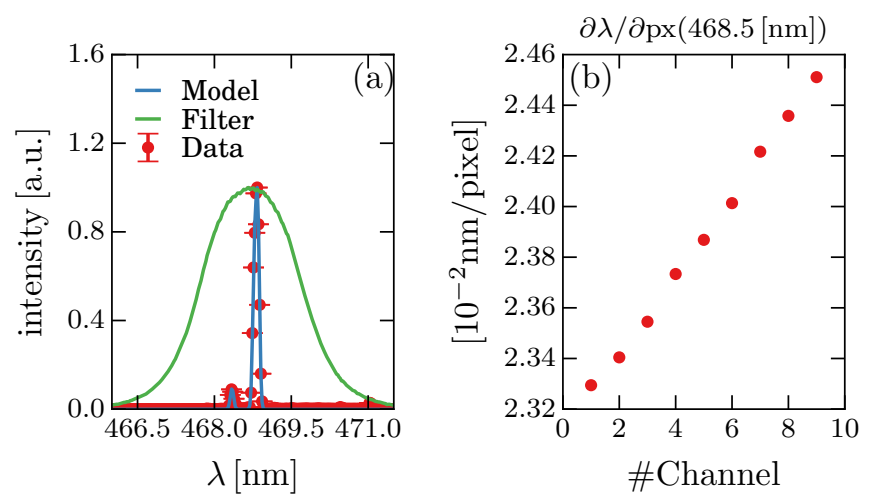

FIG. 4. (a) Forward modelling (blue) of a zirconium lamp calibration spectra (red) in the $\mathrm{He}^{2+} \mathrm{CX}$ line range. The spectra has been deconvolved from the interference filter passband characteristic (green). (b) Dispersion $\partial \lambda / \partial \mathrm{px}$ at $468.5 \mathrm{~nm}$ for the nine different slits.

spectrometer from the grating equation are given in the Appendix A. A forward model has been developed to consistently fit together the calibration spectra of different lamps in the wavelength ranges centered on the $\mathrm{CX}$ emissions of $\mathrm{He}^{2+}, \mathrm{B}^{5+}, \mathrm{N}^{7+}$ and $\mathrm{C}^{6+}$ using the previous quantities as free parameters. The reference wavelength for the calibration lamps have been taken from the NIST database ${ }^{15}$. In the forward model, parameters for the instrument function of every channel and for the intensity of every calibration line have been included to better reconstruct the calibration spectra. An example of the calibration spectra of a zirconium lamp and its model for one channel centered at the $\mathrm{He}^{2+} \mathrm{CX}$ line is shown in figure 4a. Note that every spectrometer slit can be considered as an independent spectrometer with a different opening angle. Therefore, for a certain wavelength the dispersion changes for every vertical slit (figure 4b). Moreover, the effect of the interference filter on the line shape has to be taken into account by deconvolving the calibration spectra with the shape of the filter passband (figure 4a). The accuracy of the calibration procedure is about $2 \mathrm{pm}$, i.e. roughly $1 \mathrm{~km} / \mathrm{s}$ at $468 \mathrm{~nm}$.

The passband curve of an interference filters exhibits temperature and angular dependences which changes with the wavelength $\frac{16}{}$. As a result, not only the intensity of the observed spectral lines changes but also their shape since the measured line is the result of the convolution with the filter passband. A standard integrating sphere (Labsphere, Model Unisource 1200) with a known spectral radiance has been used to characterize the shape and the transmission of the filters. Every channel has been connected alternatively to the 

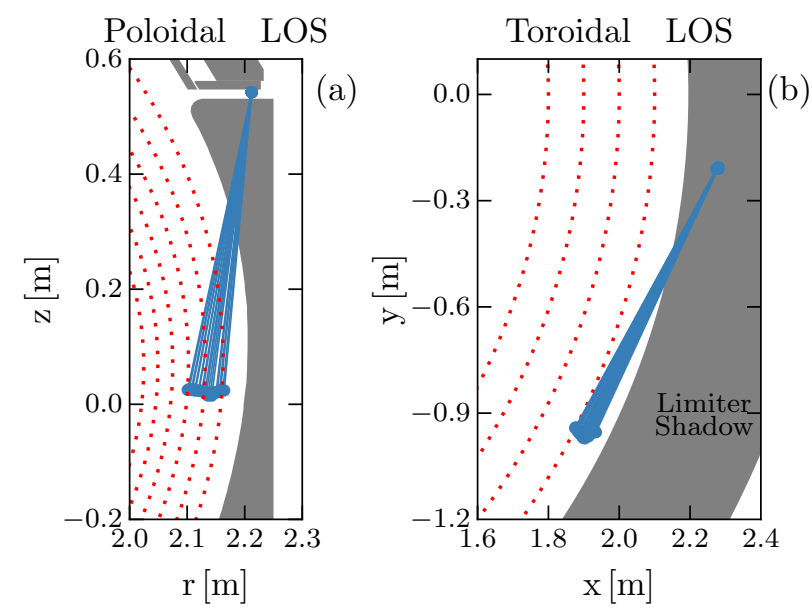

FIG. 5. Line of sight of the upgraded poloidal (a) and toroidal (b) edge charge exchange systems at ASDEX Upgrade.

Zr-lamp and to the integrating sphere before and after every discharge while the room temperature has been monitored. This procedure guaranteed the accuracy of the intensity and wavelength calibration.

\section{FIRST MEASUREMENTS}

The re-designed Czerny-Turner spectrometer using the "Spectra Kinetics" mode has successfully acquired data since the 2014 experimental campaign at ASDEX Upgrade. So far, only $\mathrm{N}^{7+}$ and $\mathrm{He}^{2+} \mathrm{CX}$ emissions were considered as possible application for fast CX measurements because their concentration in the plasma can be manipulated easily by external gas puffing ("seeding"). This turned out to be a fundamental requirement to increase the number of photons when a high repetition time is used. The spectrometer has been coupled to the edge charge exchange system which has been upgraded with additional lines of sight to increase the number of radial data points in the pedestal region. The total number of lines of sight has been increased from 16 to 50 compared to the old systems ${ }^{3}$. Moreover, the radial coverage has been reduced to roughly $6 \mathrm{~cm}$ in order to obtain fully resolved profiles of the edge ion temperature, impurity flows and density and hence of $E_{r}$, without the need of a radial plasma sweep to increase the radial resolution. The lines of sight of the refurbished poloidal and toroidal systems are shown in figure $5 \mathrm{a}$ and $5 \mathrm{~b}$. The radial resolution of the toroidal LOS is around $3 \mathrm{~mm}$ at the very edge of the plasma while it increases towards the 

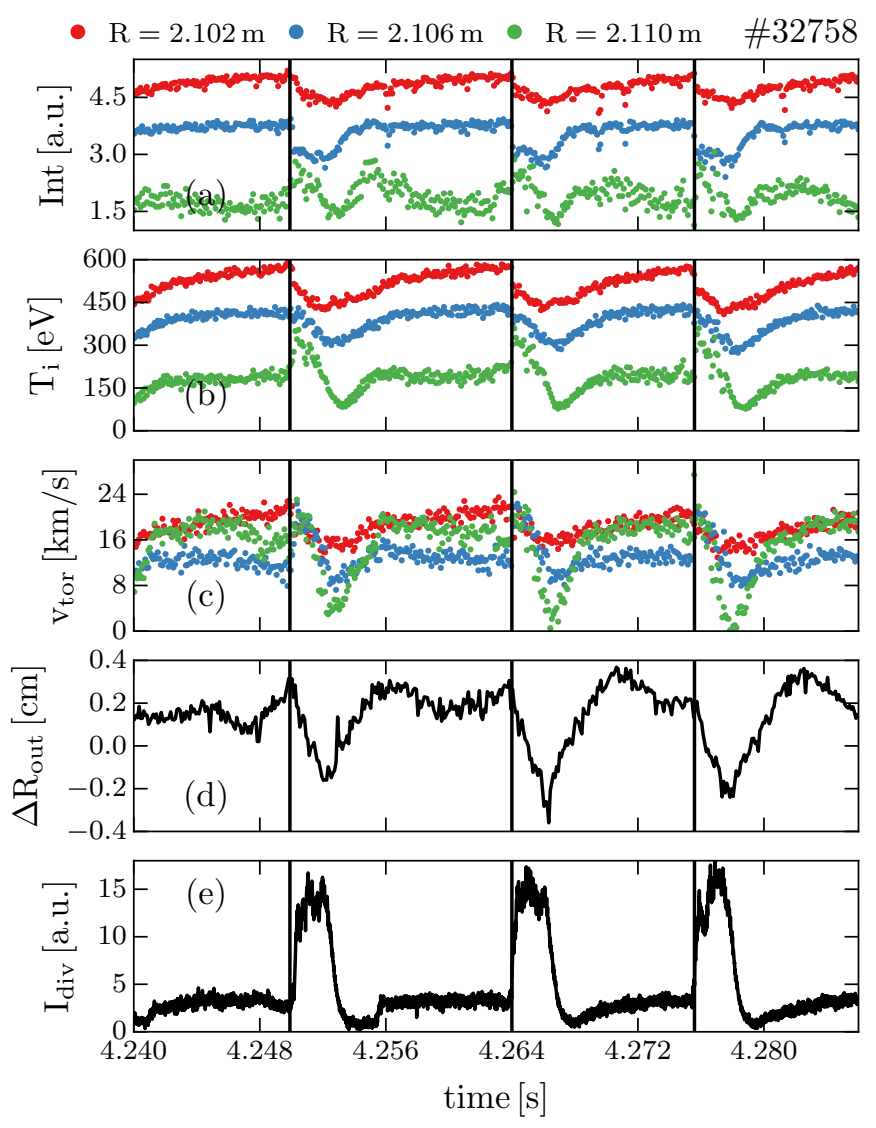

FIG. 6. Fast charge-exchange measurements of the $\operatorname{He}^{2+}(n=4 \rightarrow 3)$ line emission during edgelocalized modes in a helium plasma with a temporal resolution of 50 ps. (a) Line intensity; (b) Ion temperature; (c) Toroidal velocity; (d) Radial excursion of the plasma; (e) Divertor shunt current. The different colors indicate different radial positions.

inner part of the plasma. Since the poloidal curvature of the plasma is stronger than the toroidal one, the resolution of the poloidal system is reduced to about $5 \mathrm{~mm}$. Good signal to noise ratio measurements have been obtained for a time resolutions down to 50 us.

As an initial check of the time resolution of the system, an ELMy H-mode discharge has been examined. In figure 6, time traces of the line intensity (a), the ion temperature (b) and the toroidal velocity (c) derived from the $\operatorname{He}^{2+}(n=4 \rightarrow 3) \mathrm{CX}$ line are shown. Here, the different colors indicate different radial positions. The measurements were obtained in a helium discharge and the spectra were acquired with a temporal resolution of $50 \mu \mathrm{s}$. The profile information is compared to the radial excursion of the plasma (fig. 6d) and with the divertor shunt currents (fig. 6e) which is often used as an ELM monitor signal. Note that the data shown in figures $6 a-6 c$ are obtained by fitting single channels. Hence, the time- 
traces are shown at a fixed radial position while the plasma position changes slightly. In particularly, during the ELM crash the plasma position can change by $\Delta R_{\text {out }}=5 \mathrm{~mm}$ which is larger than the resolution of the system and therefore this effect needs to be accounted for in the flux surface mapping. The measured time traces show low statistical noise. The relative standard variation $\sigma_{r}$ is defined as the ratio of the standard deviation to the mean is calculated by binning a time window of $1 \mathrm{~ms}$, i.e. 20 measurements, during a relatively constant plasma phase before the ELM onset. The values are roughly equal to: $\sigma_{r}\left(v_{\text {tor }}\right)=4 \%$ for the toroidal velocity, $\sigma_{r}\left(T_{i}\right)=1 \%$ for the ion temperature and $\sigma_{r}$ (inte) $=3 \%$ for the line intensity. The accuracy of the measured profiles combined with the high temporal resolution open the possibility at ASDEX Upgrade to characterize in detail the evolution of the ion temperature and impurity flow profiles during ELMs and to compare the behaviour to the electron profiles similarly as in Refs. ${ }^{17}$ and $\stackrel{18}{ }$ but with better time resolution. Moreover, due to the low noise and by increasing the time resolution and the averaging time window it might be possible to monitor fluctuations of the ion temperature $\tilde{T}_{i} / \bar{T}_{i}$ and of the impurity flows $\tilde{v} / \bar{v}^{5119}$.

With the new setup, it has also been possible to detect magnetohydrodynamic activities at low frequency. An example is shown in figure 7 where the spectrogram of a ballooning coil measuring $\dot{B}_{r}$ at the low field side mid-plane of the plasma (fig. 7a) is compared to the spectrogram of $T_{i}$ measured at $\rho_{\text {pol }}=0.995$ (fig. 7b). The gray shaded areas in figure $7 \mathrm{~b}$ indicate the dead time in-between two bursts. Both measurements see a distinct mode at around $4 \mathrm{kHz}$ (highlighted in red) changing its frequency at around $3.85 \mathrm{~s}$. The mode is a global movement of the plasma which at a fixed radial position results in a fluctuation of the plasma parameters such as $T_{i}$. The detected fluctuations in $T_{i}$ induced by the mode are of the order of $2 \%$ and are strongest in the steep gradient region where a small displacement of the plasma induces the largest variation of the temperature.

Similar investigations have been attempted in N-seeded discharges in order to measure the nitrogen CX lines $\mathrm{N}^{7+}(\mathrm{n}=9 \rightarrow 8)$ with sub-ms time resolution. However, the nitrogen concentration could not be increased above roughly $3 \%$ without incurring into a disruption 20 . Hence, to reach good signal to noise ratio, the integration time could not be decreased below $200 \mu \mathrm{s}$. In this case, a spectrometer with a higher optical throughput or optics with larger etendue are necessary to further optimize the temporal resolution. 


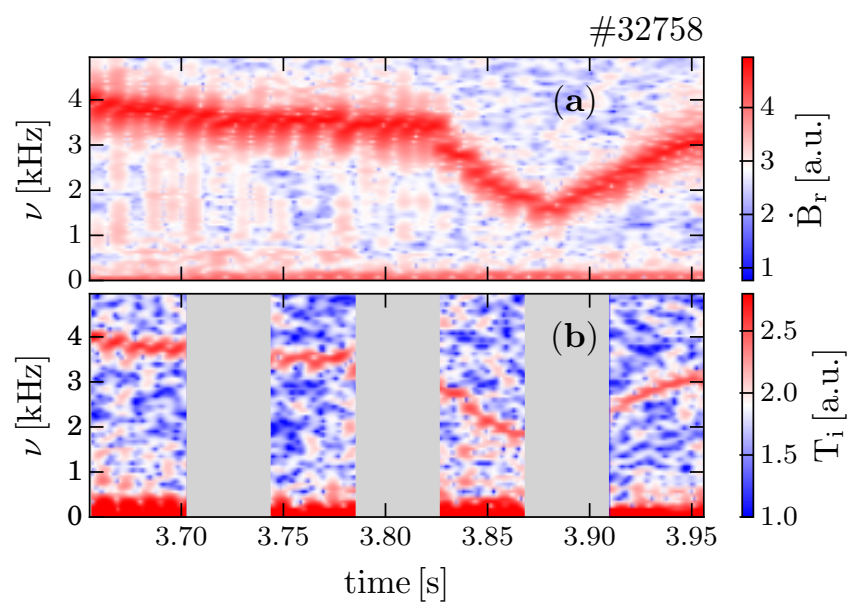

FIG. 7. (a) Spectrogram of $\dot{B}_{r}$ measured at the low field side midplane. (b) Spectrogram of $T_{i}$ at $\rho_{\text {pol }}=0.995$. The gray areas in figure (b) indicate the dead time in between two burst of the fast acquisition system.

\section{SUMMARY AND FUTURE PLANS}

In summary, a multi-channel spectrometer with about $10 \mu$ s of time resolution dedicated to edge charge exchange recombination spectroscopy has been developed at ASDEX Upgrade to characterize the fast evolution of ion temperature, impurity density and flows. A multislit design equipped with an interference filter allows for the simultaneous acquisition of multiple channel. The spectrometer has been coupled to the edge poloidal and toroidal charge exchange systems which have been refurbished with 50 new lines of sight covering a radial range of about $6 \mathrm{~cm}$. The new setup offers the possibility to measure detailed profiles during fast events without the necessity of a radial sweep of the plasma.

Good signal to noise ratio has been obtained for an integration time of $50 \mu$ s during edge localized modes measuring the $\mathrm{He}^{2+}$ charge exchange emission. The measurements achieved a statistical noise of a few percent. Hence, it is expected that using the highest temporal resolution $(10 \mu \mathrm{s})$, low frequency ion temperature and impurity flow fluctuations might be detectable.

A larger CCD sensor would allow the simultaneous acquisition of more channels while the read-out time, i.e. the acquisition dead time, would be increased. More spectrometer channels can be employed by increasing the spectrometer dispersion but this might influence the accuracy of the velocity measurements. While the working principle of the modified 


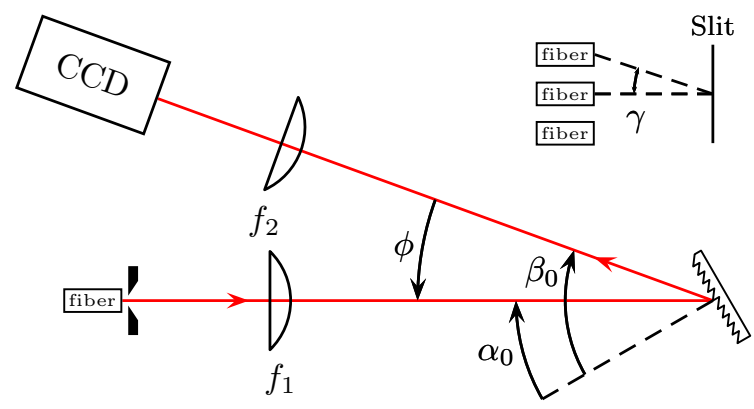

FIG. 8. Schematic of a Czerny-Turner spectrometer with reflective grating

Czerny-Turner spectrometer has been successfully tested, different setups need to be investigated to relatively optimize the number of acquirable channels, the acquisition dead time and the measurement accuracy. The optimum solution might depend on the investigated phenomena.

\section{Appendix A: Determination of the wavelength axis of a Czerny-Turner spectrometer}

The wavelength axis of a Czerny-Turner spectrometer adopting reflective gratings (see figure 8) is determined by the grating equation ${ }^{14}$ :

$$
n g \lambda=\cos \gamma(\sin \alpha+\sin \beta)
$$

where $n$ is the diffraction order, $g$ the number of grooves $/ \mathrm{mm}$ of the grating, $\lambda$ the wavelength, $\gamma$ the vertical angle of an off-axis ray, $\alpha$ and $\beta$ the incident and the diffraction angle from the grating perpendicular axis, respectively. Note that the definition of the angles $\alpha$ and $\beta$ follows the mathematical formalism, i.e. counter-clockwise angles are positive while clockwise angles are negative. In the example shown in figure 1 both $\alpha$ and $\beta$ are negative. In this way, the spectrometer opening angle is determined as $\phi=\alpha_{0}-\beta_{0}$ with $\phi$ positive. The horizontal distance from the optical axis on the chip plane $x_{2}$ are defined as $\delta \beta \approx \tan \delta \beta=x_{2} / f_{2}, f_{2}$ being the focal length of the focussing lens. Assuming small angles $\gamma \ll 1$ and $\delta \beta \ll \beta_{0}$, equation A1 can be approximated by a Taylor expansion for two variables $\gamma$ and $\beta$ around $A=\left(\gamma=0, \beta=\beta_{0}\right)$ for an entrance slit aligned to the optical axis, i.e. $\alpha=\alpha_{0}$. Since the size of the camera chip $(8.2 \mathrm{~mm} \times 8.2 \mathrm{~mm})$ is relatively small compared to the focal lengths of the lenses $(280 \mathrm{~mm}$ and $180 \mathrm{~mm}$ ), the equation A1 has 
been approximated only up to second order:

$$
\lambda=\lambda_{0}+\left.\frac{\partial \lambda}{\partial \gamma}\right|_{A} \gamma+\left.\frac{\partial \lambda}{\partial \beta}\right|_{A} \delta \beta+\left.\frac{\partial^{2} \lambda}{\partial \gamma^{2}}\right|_{A} \frac{\gamma^{2}}{2}+\left.\frac{\partial^{2} \lambda}{\partial \gamma \partial \beta}\right|_{A} \gamma \delta \beta+\left.\frac{\partial^{2} \lambda}{\partial \beta^{2}}\right|_{A} \frac{\delta \beta^{2}}{2}+\cdots
$$

with the single contributions:

$$
\begin{aligned}
\lambda_{0} & =\frac{\sin \alpha_{0}+\sin \beta_{0}}{n g}, \\
\left.\frac{\partial \lambda}{\partial \gamma}\right|_{A} & =0 \\
\left.\frac{\partial \lambda}{\partial \beta}\right|_{A} & =\frac{\cos \beta_{0}}{n g} \\
\left.\frac{\partial^{2} \lambda}{\partial \gamma^{2}}\right|_{A} & =-\lambda_{0} \\
\left.\frac{\partial^{2} \lambda}{\partial \gamma \partial \beta}\right|_{A} & =0 \\
\left.\frac{\partial^{2} \lambda}{\partial x_{2}^{2}}\right|_{A} & =-\frac{\sin \beta_{0}}{n g} .
\end{aligned}
$$

Note that the first and the second order dispersion (Eq.s A5 and (A8) do not contain any $\gamma$ dependence and the mixed term (A7) is equal to zero. Hence the first and second order dispersions are the same for every channel. These are calculated from $\lambda_{0}$ and $f_{2}$ since the angles $\alpha_{0}$ and $\beta_{0}$ can be calculated from (A3) knowing the relation $\phi=\alpha_{0}-\beta_{0}$. The dependence of the vertical position of the fiber is determined only by the second order expansion on $\gamma$ and it has the typical parabolic dependence on the vertical angle $\sim \gamma^{2}$ (Eq. (A2) ) which given $\gamma \ll 1$ can be approximated as $\gamma \approx \tan \gamma=y_{2} / f_{2}$ where $y_{2}$ is the vertical position on the camera chip with respect to the optical axis. Note that the vertical position of the optical axis does not always correspond to the middle of the chip. Hence, $y_{2}$ needs to be corrected by a vertical shift $\delta_{h}$. Additionally, possible deviation of every single fiber from the horizontal position of the optical axis are accounted by correcting $x_{2}$ with a shift $\delta x$. The resulting equation used in this work for the wavelength at a certain position $\left(x_{2}, y_{2}\right)$ on the chip is:

$$
\lambda=\lambda_{0}+\frac{\cos \beta_{0}}{n g} \frac{x_{2}-\delta_{x}}{f_{2}}-\lambda_{0}\left(\frac{y_{2}-\delta_{h}}{f_{2}}\right)^{2}-\frac{1}{2} \frac{\sin \beta_{0}}{n g} \frac{\left(x_{2}-\delta_{x}\right)^{2}}{f_{2}^{2}}
$$

where $\beta_{0}=\frac{\phi}{2}-\arcsin \left(\frac{n g \lambda_{0}}{2 \cos (\phi / 2)}\right) 1$ and $\alpha_{0}=\phi+\beta_{0}$. Consequently, the free parameters of the wavelength calibration are: $\lambda_{0}, f_{2}, \delta_{h}$ and $\delta_{x}$.

\footnotetext{
${ }^{1}$ From Eq. A3 using the trigonometric identity: $\sin x+\sin y=2 \sin ((x+y) / 2) \cos ((x-y) / 2)$.
} 


\section{ACKNOWLEDGMENTS}

This work has been carried out within the framework of the EUROfusion Consortium and has received funding from the Euratom research and training programme 2014-2018 under grant agreement No 633053. The views and opinions expressed herein do not necessarily reflect those of the European Commission.

\section{REFERENCES}

${ }^{1}$ R. Fonck, D. Darrow, and K. Jaehnig, Phys. Rev. A 29, 3288 (1984).

${ }^{2}$ W. Mandl, R. C. Wolf, M. G. von Hellermann, and H. P. Summers, Plasma Phys. and Contr. Fusion 35, 1373 (1993).

${ }^{3}$ E. Viezzer, T. Pütterich, R. Dux, R. M. McDermott, and the ASDEX Upgrade Team, Rev. Sci. Instrum. 83, 103501 (2012).

${ }^{4} \mathrm{R}$. McDermott et al., in preparation (2016).

${ }^{5}$ H. T. Evensen, R. Durst, R. J. Fonck, and S. F. Paul, Rev. Sci. Instrum. 66, 845 (1995).

${ }^{6}$ D. M. Thomas, K. H. Burrell, R. J. Groebner, P. Gohil, D. Kaplan, C. Makariou, and R. P. Seraydarian, Rev. Sci. Instrum. 68, 1233 (1997),

${ }^{7}$ S. Kobayashi, A. Sakasai, Y. Koide, Y. Sakamoto, Y. Kamada, T. Hatae, N. Oyama, and Y. Miura, Journal of Plasma and Fusion Research 79, 1043 (2003).

${ }^{8}$ D. Craig, D. J. Den Hartog, D. A. Ennis, S. Gangadhara, and D. Holly, Rev. Sci. Instrum. 78, 013103 (2007).

${ }^{9}$ I. U. Uzun-Kaymak, R. J. Fonck, G. R. McKee, N. Schoenbeck, D. Smith, G. Winz, and

Z. Yan, Rev. Sci. Instrum. 81, 10D714 (2010), http://dx.doi.org/10.1063/1.3479114.

${ }^{10}$ Leica, http://us.leica-camera.com/.

${ }^{11}$ Princeton Instruments, http://www.princetoninstruments.com/.

${ }^{12}$ N. J. Conway, P. G. Carolan, J. McCone, M. J. Walsh, and M. Wisse, Rev. Sci. Instrum. 77, 10F131 (2006), http://dx.doi.org/10.1063/1.2354309.

${ }^{13}$ R. E. Bell and R. Feder, Rev. Sci. Instrum. 81, 10D724 (2010), http://dx.doi.org/10.1063/1.3485027.

${ }^{14}$ R. E. Bell, Rev. Sci. Instrum. 75, 4158 (2004).

${ }^{15}$ National Institute of Standards and Technology (NIST), http://www.nist.gov/. 
${ }^{16}$ D. Morelli, Interference Filter Handbook: A Guide for Specifying Optimum Filter Performance (Optical Coating Laboratory, 1991).

${ }^{17}$ E. Viezzer, T. Pütterich, G. Conway, R. Dux, T. Happel, J. Fuchs, R. McDermott, F. Ryter, B. Sieglin, W. Suttrop, M. Willensdorfer, E. Wolfrum, and the ASDEX Upgrade Team, Nucl. Fusion 53, 053005 (2013).

${ }^{18}$ M. R. Wade, K. H. Burrell, J. T. Hogan, a. W. Leonard, T. H. Osborne, P. B. Snyder, and D. Coster, Phys. Plasmas 12, 056120 (2005).

${ }^{19}$ I. U. Uzun-Kaymak, R. J. Fonck, and G. R. McKee, Rev. Sci. Instrum. 83, 10D526 (2012),

${ }^{20}$ M. Bernert et al., submitted to Nuclear Materials and Energy (2016). 